\title{
Área de Vertebrados do Museu Paraense Emílio Goeldi
}

\author{
F. C. Novaes (") \\ O. R. Cunha (")
}

\begin{abstract}
Resumo
Breve relato das atividades da área de Vertebrados do Museu Paraense Emilio Goeldi quando passou em janeiro de 1955 a ser administrado pelo Conselho Nacional de Pesquisas. As principais coleções científicas são a de mamiferos com cerca de 9.000 exemplares, Aves com 37.718 e Herpetologia 35.000 exemplares.
\end{abstract}

Por convênio realizado em 7 de dezembro de 1954, entre o Estado do Pará e o Instituto Nacional de Pesquisas da Amazônia (INPA), foi a direção científica e a administração do Museu Paraense Emílio Goeldi entregue a esse órgão a partir de 01 de janeiro de 1955.

Das quatro Divisões de pesquisas em que - Museu Goeldi era então estruturado, uma delas a Divisão de Zoologia foi mantida até fevereiro de 1977, quando foi desdobrada em dois Departamentos o de Vertebrados e de Invertebrados. No ano de 1980 os dois Departamentos foram anexados ao Departamento de Biologia.

De 1955 até os dias atuais as coleções científicas de Vertebrados do Museu receberam apoio das diferentes diretorias do INPA e Museu. Seu patrimônio foi substancialmente enriquecido e as pesquisas retomadas, pois a muito tempo estavam paralisadas por falta de pesquisadores.

A partir da data do convênio até meados de 1973 as coleções de Vertebrados encontravam-se precariamente instaladas em diversas salas e diferentes prédios do Museu o que dificultava seu manuseio e consulta, além de serem prédios muito velhos e de difícil manutenção.

Em 1973, quando o Dr. Paulo de Almeida Machado dirigia o INPA foram construídos novos pavilhões. Assim, foi possivel reuní-las em dois prédios, abrangendo atualmente uma área construída, superior a 800 metros quadrados.

São as seguintes as coleções de estudo na área de Vertebrados do Museu Goeldi.

\section{MASTOZOOLOGIA}

As coleções científicas de mamíferos do Museu, remontam do início do século quando foram iniciadas principalmente por Emílio Goeldi e Gottfried Hagmann.

Todos os grandes grupos da área amazônica acham-se representados, destacando-se entretanto a dos primatas, quirópteros e roedores. Esta coleção permite uma variedade de estudos em sistemática, biometria e evolução. O mastozoólogo contratado pelo Conselho $\mathrm{Na}$ cional de Pesquisas em 1955 para recuperar as coleções foi Cory T. Carvalho que exerceu suas funções até 1959. Desta data até os dias atuais não foi possível contratar nenhum pesquisador que desse continuidade aos estudos de mastozoologia no Museu.

Os principais trabalhos sobre mamiferos publicados nos periódicos do Museu Goeldi foram de Cory T. Carvalho (1957), Avila-Pires (1958) e Piccinini (1974).

O estudo da mastozoologia no Brasil vem sendo feito sem continuidade por falta de cursos de especialização. Um dos mais reputados mastozoólogo patrício Prof. Dr. J. Moogen já em 1952 no prefácio de seu livro Roedores do Brasil adverte sobre a carência de pesquisadores na área e até nos dias atuais, suas observações continuam vigentes.

Pesquisadores estrangeiros e brasileiros têm utilizado as coleções de mamíferos do Museu Goeldi tais como: R. Wetzel, Univer-

(*) Museu Paraense Emílio Goeldi, Belém. 


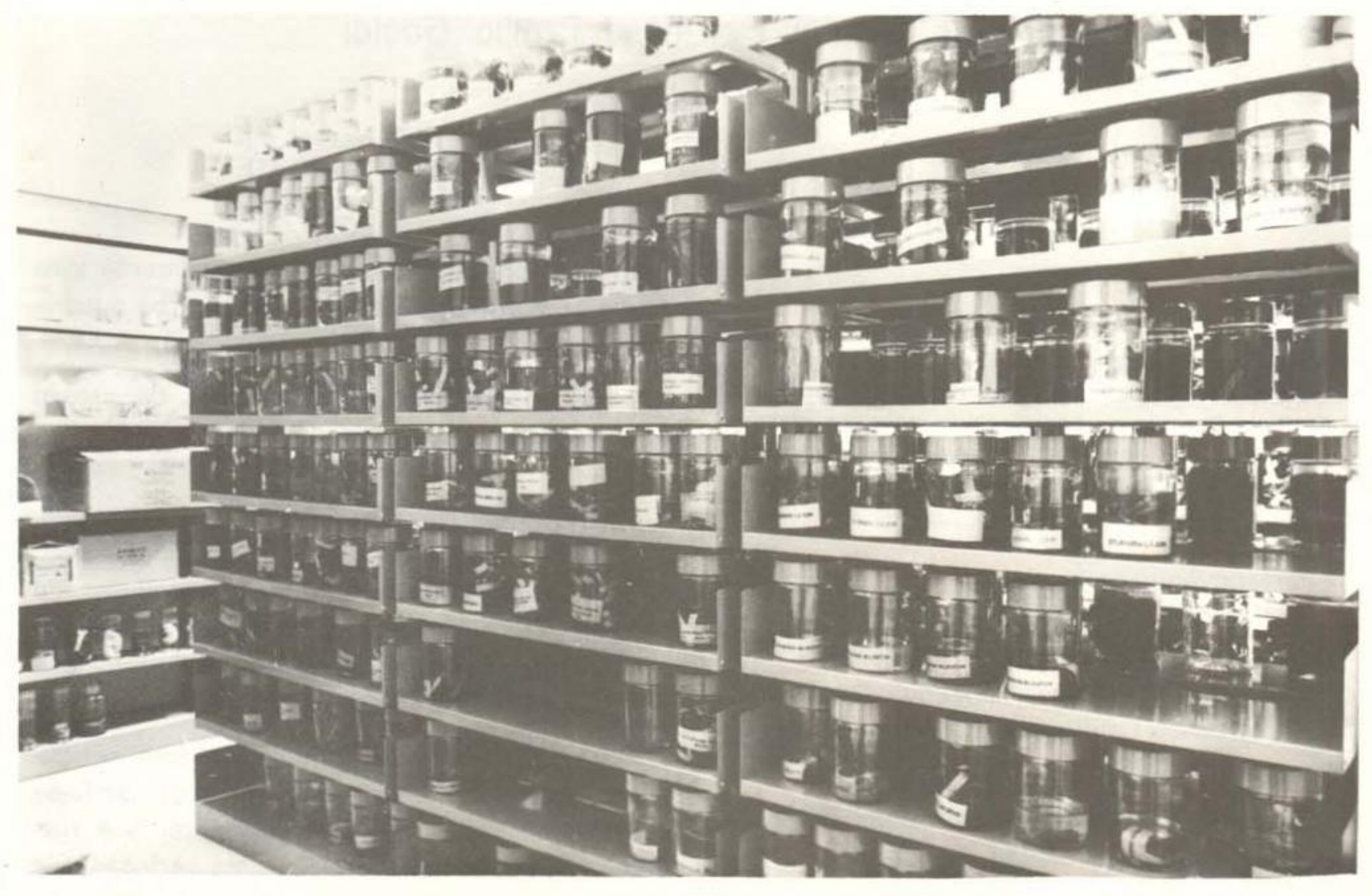

Fig. 1 - Vista geral das coleçōes em meio liquido de Quirópteros.

sity of Connecticut, USA ( $1975,1978,1980)$; P. Hershkovitz, Field Museum of natural History. Chicago (1979); V. A. Taddei, Universidade Estadual Paulista, São José do Rio Preto-SP (1978).

Atualmente estão registrados mais de 9000 peles e crânios de Mamíferos na coleção de estudo. A função de curadoria está a car. go do pesquisador F. C. Novaes.

\section{ORNITOLOGIA}

O início da coleção ornitológica data do tempo de Emílio Goeldi, quando veio ao Pará para reorganizar o Museu no final do século XIX. Tomou grande impulso quando em 1905 , Emilia Snethlage passou a ter a seu cargo a curatoria da coleção. Em 1955, quando o Conselho Nacional de Pesquisas passou a administrar o Museu, contava a coleção com cerca de 14.000 exemplares de estudo registrados. Atualmente, a coleção registra o seguinte nú- mero de exemplares: Aves (peles) 33.383; Aves (esqueletos) 3.065; Aves (meio líquido) cerca de 920; ninhos de aves (aproximadamente) 200; ovos (aproximadamente) 150 .

Os estudos de ornitologia foram reiniciados quando F.C. Novaes passou a ter a seu cargo a coleção ornitológica a partir de abril de 1955. As linhas de pesquisas desenvolvidas foram em vários setores, tais como, a biologia do comportamento (autoecologia) desenvolvidas principalmente por C.T. Carvalho (19571960). Esses estudos foram realizados no parque botânico do Museu e na reserva de flores. tas do antigo Instituto Agronômico do Norte (IPEAN), hoje pertencente à EMBRAPA.

As pesquisas faunísticas foram desenvolvidas por F.C. Novaes (1957-1980). Várias regiōes foram pesquisadas, tais como, no Território do Amapá, Acre (rio Juruá), rio Xingu, rio Acará, região leste do Pará, rio Fresco, rio $\mathrm{Pa}$. ru de Leste, rio Aripuană, rio Negro, etc . 
Estas pesquisas tiveram como objetivo o levantamento preliminar de diferentes tipos de comunidades biológicas nas áreas estudadas.

Durante os anos de 1965 a 1970 o setor de Aves colaborou com o Instituto Evandro Chagas da Fundação SESP-Belém no estudo da ecologia das aves, possivelmente reservató. rios naturais de numerosos arbovirus.

\section{HERPETOLOGIA}

Durante os últimos vinte anos os estudos de herpetologia da Amazônia tiveram grande desenvolvimento no Museu Goeldi, devidas ao pesquisador Osvaldo Rodrigues da Cunha. O primeiro trabalho sobre lagartos da região, na nova fase de recuperação do Museu foi publicado em 1958 (Cunha, 1958). Mas o estudo que marcou o ponto de desenvolvimento da herpetologia no Museu Goeldi foi o de Cunha (1961).

Até 1965 , as coleções de répteis do Museu Emílio Goeldi eram escassas e não iam além de 1.000 exemplares. Faltava um maior incentivo, auxiliares e uma melhor coordenação de trabalhos de campo. Para proporcionar um desenvolvimento acelerado dos estudos herpetológicos e aumentar intensiva e sucessivamente as coleções em várias regiões da Amazônia, o pesquisador Osvaldo Cunha reorganizou o setor de Herpetologia, assentada num esquema prático e científico, no ano de 1965 com o apoio integral do então Diretor Dalcy de Oliveira Albuquerque. Foi convocado como auxiliar de pesquisas no novo setor o $\mathrm{Sr}$. Francisco Paiva do Nascimento, o qual orientado dentro de um programa, especializou-se na subordem dos ofídios (cobras).

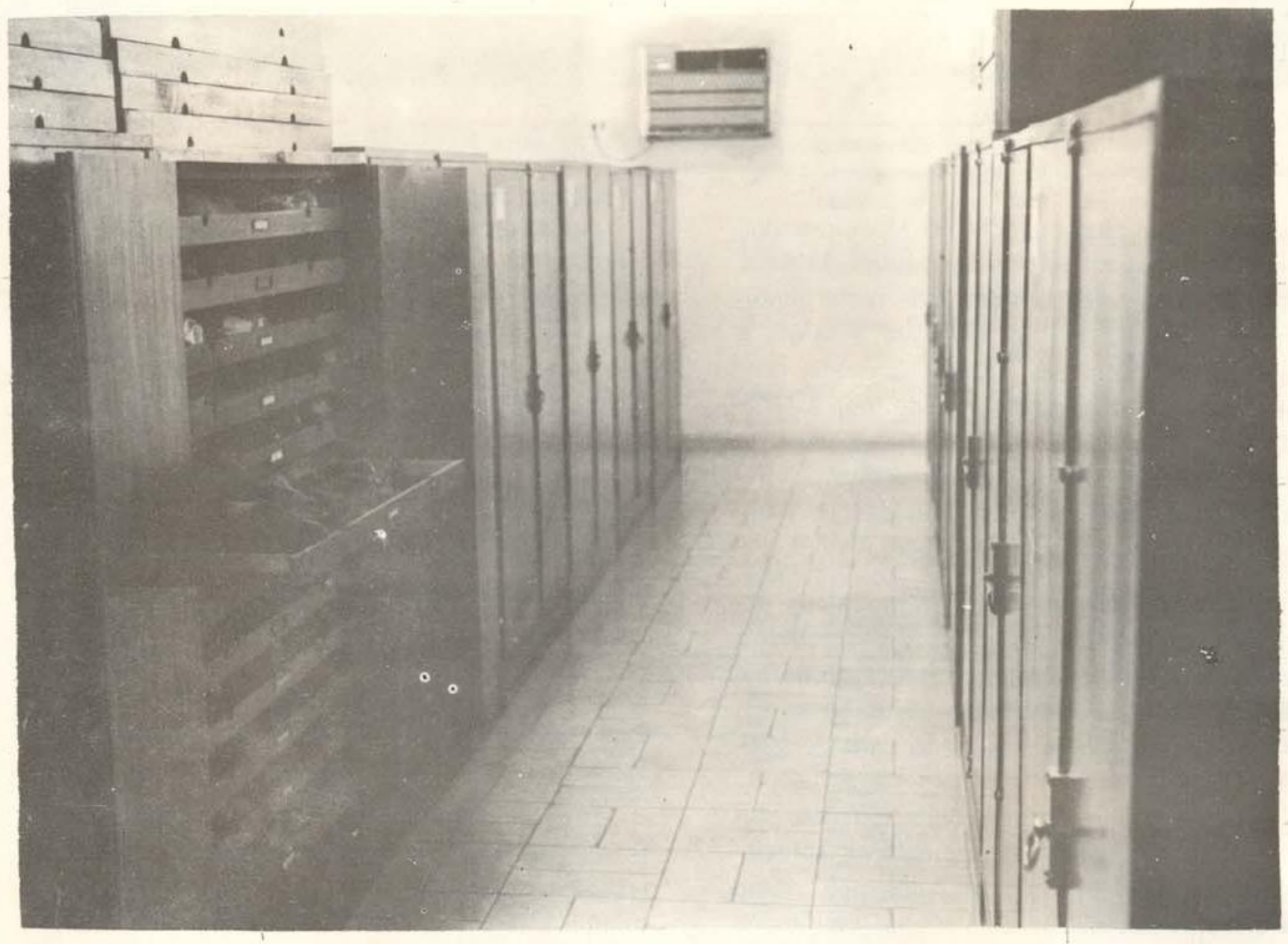

Fig. 2 - Vista parcial das coleçōes de peles de Mamíferos e Aves. 


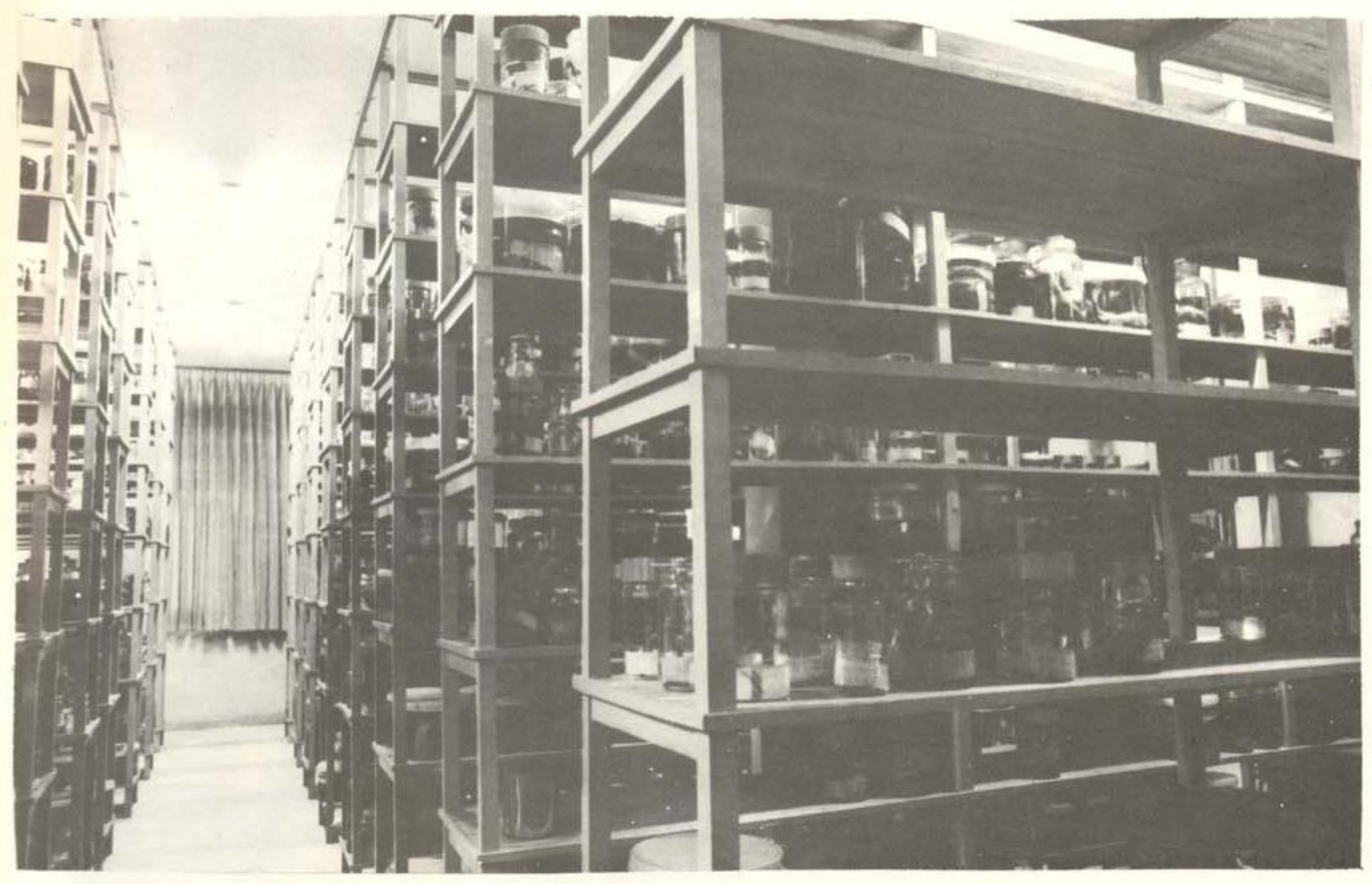

Fig. 3 - As coleçōes de répteis e anfibios do setor de Herpetologia.

O setor de Herpetologia do Museu, a única especializada no estudo dos répteis da Amazônia, e atualmente uma das três mais importantes do Brasil, tem por objetivo a coleta e o estudo científico dos ofídios, lagartos, quelônios, crocodilianos e anfíbios, de toda a Amazônia e em alguns casos de outras partes do Brasil. Um dos objetivos básicos das pesquisas é aquela que são desenvolvidas no campo, estudando a ecologia das espécies.

No espaço de onze anos os trabalhos desenvolveram-se rapidamente com excursões a algumas importantes áreas de interesse no estudo dos répteis. Coletas intensivas foram então levadas a cabo, aumentando consideravelmente o número de espécimes conservados. Foi iniciado catálogos para cada um dos grupos de répteis, sendo os exemplares selecio. nados, etiquetados e identificados pelo nome científico e procedência. Alguns resultados das pesquisas de campo foram elaborados $\mathrm{e}$ paulatinamente estão sendo publicados.
A partir de 1971 foi estabelecido projeto a longo prazo objetivando a coleta intensiva de espécimes e ecologia.

- Projeto levantamento dos ofídios e lagartos da região leste do Pará. Esta região que engloba toda a zona bragantina, do litoral e as bacias dos rios Guamá e Gurupi no Pará, perfaz uma área de cerca de $45.000 \mathrm{~km}^{2}$. Por mais paradoxal que pareça, a região mais conhecida e habitada no Pará, quase uma extensão de Belém, era até então uma das menos conhecidas com relação aos répteis. Algumas poucas referências existem, muito esparsas, feitas por Florêncio Gomes do Instituto Butantan, São Paulo, em 1918 sobre alguns exemplares na época pelo pessoal do Museu Goeldi.

Durante mais de quatro anos a região les. te do Pará foi percorrida e estudada. Estabeleceram-se vinte e dois postos de coletas de ofídios e lagartos, em vários locais tendo por base as várias comunidades vegetais que ai se encontram, isto é, mata primária (quase toda 
devastada), campos naturais, roçados, capoeiras em vários estágios e várzeas de rios. Para que o trabalho de campo fosse o mais completo possível, os pesquisadores solicitaram a cooperação imprescindivel dos habitantes locais. No laboratório os estudos desenvolveram-se aceleradamente para a seleção do material e identificação taxonômica das espécies. Com o término dos trabalhos de campo em 1975 , o resultado foi o mais surpreendentemente possivel. Cerca de 20.000 exemplares de ofídios, lagartos, sapos, rãs e tartarugas foram coletados nesse espaço de tempo.

Vários trabalhos já foram publicados sobre os ofídios e lagartos da região leste, salientando-se entre outros os seguintes : Cunha \& Nascimento, 1973, 1975, 1976, 1978; Cunha, 1977.

Atualmente o setor de Herpetologia do Museu conta em suas coleções cerca de 35.000 exemplares de répteis e anfíbios.

Terminando este breve relato sobre as atividades científicas do Museu Goeldi desde que sua administração passou a órbita do CNPq transcreveremos as palavras do eminente Prof. Dr. Lauro Travassos (já falecido), quando em 1958 trabalhou na Divisão de Zoologia do Museu (Travassos \& Kloss, 1958) :

Cumpre salientar a nova organização do tradicional Museu Goeldi, atualmente sob os auspícios do Conselho Nacional de Pesquisas. Tudo o que foi possível salvar do soberbo material que lá ainda existia foi preservado pelo atual Diretor.

O Conselho Nacional de Pesquisas teve o mérito de salvar do abandono e aniquilamento completo a tradicional instituição de merecimento internacional, que ś o Museu Emílio Goeldi e que atualmente representa ótima base para estudo dos diversos ramos das Ciências Naturais na Amazônia.

\section{SUMMARY}

A short account of the research activities of the Museum Goeldi, section of Vertebrates during the period from January of 1955 until present data when the Conselho Nacional de Pesquisas took under his charge its administration. The main research collections are mammals with 9.000 specimens, birds 37.718 specimens and reptiles, snakes and amphibianswith about 35.000 specimens.

\section{REFERENCIAS BIBLIOGRAFICAS}

AVILA-PIRES, F.D. de

1958 - Mamíferos colecionados nos arredores de Belém do Pará. B. Mus. Pa. Emílio Goeldi, n. ser. Zool., Belém, 19: 1-6.

CARVALHO, C.T. de

1957 - Notas ecológicas sobre Volatinia jacarina. B. Mus. Pa. Emílio Goeldi, n. ser. Zool., Belém, 2: 1-10.

1957 - Alguns mamíferos do Acre ocidental. B Mus. Pa. Emilio Goeldi, n. ser. Zool., Belém, 6: 1-22.

1957 - Relações biológicas entre Columbigallina paserina e C. talpacoti (Aves, Columbidae). B. Mus. Pa. Emílio Goeldi, n. ser. Zool., Belém, 7: 1-15. il.

1958 - Sobre alguns mamíferos do sudeste do $\mathrm{Pa}$ rá. Arq. Zool., São Paulo, 11 5): 121-132

1960 - Das visitas de morcegos às flores (Mammalia, Chiroptera). An. Acad. Bras. Ciênc., Rio de Janeiro, 32 (3/4): 359-377.

1960 - Comportamento de Myozetetes cayanensis e notas biológicas sobre espécies afins. (Passeres, Tyrannidae). Papéis. Avulsos. Dep. Zool., São Paulo, 14 (15): 122-132.

CUNHA, O.R. da

1958 - 1. Lacertílios da Amazônia. Sobre a ocorrência do gênero Bachia Gray, 1845 na Amazônia brasileira. B. Mus. Pa. Emilio Goeldi, n. ser. Zool., Belém, 11: 1-12. il.

1961 - II. Lacertílios da Amazônia. Os lagartos da Amazônia brasileira, com especial referência aos representados na coleção do Museu Goeldi. B. Mus. Pa. Emílio Goeldi, n. ser. Zool., Belém, 39: 1-189.

1977 - Lacertílios da Amaônia VI. Uma nova espécie de lagarto (Colobosaura landii) da região do leste do Pará (Lacertilia, Teiidae). B. Mus. Pa. Emílio Goeldi, n. ser. Zool. Belém, 86: 1-13. il.

CUNHA, O.R. da \& NASCIMENTO, F.P.

1973 - Ofídios da Amazônia IV. As cobras corais (gênero Micrurus) da região leste do Pará (Ophidia, Elapidae). Nota preliminar. In: SIMŐES, Mário F., ed. O Museu Goeldi no ano do Sesquicentenário. Publ. Avulsas Mus. Pa. Emílio Goeldi, Belém, 20: 273-286

1975 - Ofídios da Amazônia VII. As serpentes peçonhentas do gênero Bothrops (Jararacas) e Lachesis (Surucucu) da região leste do $\mathrm{Pa}$ rá. (Ophidia, Viperidae). B. Mus. Pa. Emilio Goeldi, n. ser. Zool., Belém, 83: 1-42. il.

1976 - Ofídios da Amazônia IX. O gênero Liophis Wagler 1830 na região leste do Pará. (Ophidia, Colubridae). B. Mus. Pa. Emílio Goeldi, n. ser. Zool., Belém, 85: 1-32. il. 
1978 - Ofídios da Amazônia X. As cobras da re gião leste do Pará. Publ. Avulsas Mus. Pa Emílio Goeldi, Belém, 31: 1-128. il.

HERSHKOVITZ, P

1977 - Living new worls monkeys (Platyrrhini) Universidade Chicago Press, 1117 p. il.

MOOGEN, J.

1952 - Os Roedores do Brasil Rio de Janeiro, Inst. Nac. do Livro, 214 p. il.

NOVAES, F.C.

1957 - Contribuição à ornitologia do noroeste do Acre. B. Mus. Pa. Emílio Goeldi, n, ser Zool., Belém, 9: 1-30.

1968 - Variação geográfica em Platyrhinchus saturatus Salvin \& Godman (Aves, Tyrannidae). Rev. bras. Biol., Rio de Janeiro, 28 (2): 115-119.

1969 - Análise ecológica de uma avifauna da região do rio Acará, Estado do Pará. B. Mus. Ma. Emílio Goeldi, n. ser. Zool., Belém, 69: 1-52. il.

1970 - Distribuição ecológica e abundância das aves em um trecho da mata do baixo rio Guamá. B. Mus. Pa. Emílio Goeldi, n. ser Zool., Belém, 71: 1-54. il.

1973 - Aves de uma vegetação secundária na foz do Amazonas. Publ. Avulsas Mus. Pa. Emílio Goeldi, Belém, 21: 1-88. il.

1974 - Onitologia do Território do Amapá I. Publ. Avulsas Mus. Pa. Emílio Goeldi, Belém, 25. 1-121.

1976 - As aves do rio Aripuanã, Estados de Mato Grosso e Amazonas. Acta Amazonica, Manaus, 6 (4) Suplemento: 61-85.

1978 - Ornitologia do Território do Amapá II. Publ. Avulsas Mus. Pa. Emílio Goeldi, Belém, 29: 1-75.

1980 - Observações sobre a avifauna do alto curso do rio Paru de Leste, Estado do Pará. B. Mus. Pa. Emílio Goeldi, n. ser. Zool., Belém, 100: 1-58.
NOVAES, F.C. \& CARVALHO, C.T. de

1957 - Observações sobre a nidificação de Glaucis hirsuta (Trochilidae, Aves). B. Mus. Pa. Emilio Goeldi, n. ser. Zool., Belém, 1: 1-11. il.

NOVAES, F.C. \& PIMENTEL, T.

1973 - Observações sobre a avifauna do campos de Bragança, Estado do Pará. In: SIMŌES, Mário F. ed. O Museu Goeldi no ano do Sesquicentenário. Publ. Avulsas Mus. Pa. Emilio Goeldi, Belém, 20: 229-246.

PICCININI, R.S.

1974 - Lista provisória dos quirópteros da coleção do Museu Paraense Emílio Goeldi. B. Mus. Pa. Emílio Goeldi, n. ser. Zool., Belém, 77: $1-32$.

TADDEI, V.A. et alii.

1978 - Notas sobre Lionycteris e Lonchophylla nas coleções do Museu Paraense Emílio Goeldi (Mammalia, Chiroptera, Phyllostomidae). B. Mus. Pa. Emílio Goeldi, n. ser. Zool., Belém, 92: 1-14. il.

TRAVASSOS, L. \& KLOSS, G.R.

1958 - Relatório da excursão do Instituto Oswaldo Cruz e Museu Nacional à Belém-Pará de dois a dezessete de março de mil novecentos e cincoenta e oito. B. Mus. Pa. Emílio Goeldi, n. ser. Zool., Belém, 15: 1-19. il.

WETZEL, R.M.

1975 - The species of Tamandua Gray (Edentata, Myrmecophagodae). Proc. biol. soc. Washington, 66 (11): 95-112.

1979 - The subgenera and species of Long-nosed Armadillos, Genus Dasypus L. In: EISENBERG, J.F. Vertebrate Ecology in Northern Neotropics, Washington, Smithsonian Press, p. 43-63.

1980 - Revision of the Naked-tailed Armadillos Genus Cabassous Mc Murtrie. Ann. Carnegie Mus., Pittsburgh, 49 (20): 323-357. 